\title{
$\mathfrak{U} \mathfrak{u}$ ber Borrebe zur erften $\mathfrak{A}$ uflage.
}

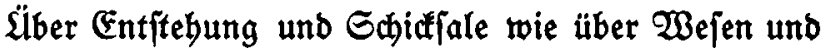

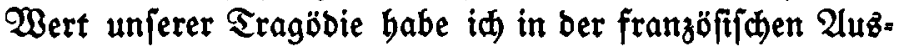
gabe berjelben (2. 2uflage, Gtraß̈burg, Trübner 1902) baz Nötige gefagt und tann baber, um mid) nicht in mand)em bier zu wieberbolen, einfach bortbin verweifen. . .

Dieje meine Berbeutjhung habe id mir nidjt etwa nur alz literarifhes Probuft vorgeftellt, fonbern gleich vom erften Slugenblide an audh bie beutiche $\mathfrak{B u ̈ b n e ~ f e h r ~}$ ernjtlich bafür inz 2luge gefaj̧t. Ia) würbe es um biejer felbjut willen betlagen, wenn inmitten bez reiden Lebens, Daz unjer Sheater neuerbingz entfaltet, allez baz von Geiftesłafaft, Soerzenżglut unb Geelengröß̄e, raaz Gobineau

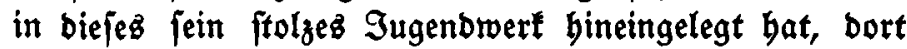
leine Seimftätte finden follte.

aber ez iff bas wohl aud faum zu fürdhten. Qeben= big genug legt Das Orama jelbjt fajt in jebem feiner Worte 3eugniz bafür ab, baß hier ein Großjer rebet, unb wenn fids nur bie rechten Darfteller für beffen bid)= terifhe $\mathfrak{d} b$ fiditen finben, fo ericheinen jeinem flammenden

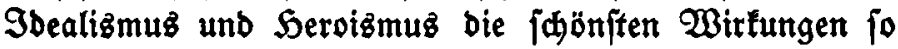
gut wie gefichert.

Sierüber alfo wäre faum erf́t nod ein $\mathfrak{W o r t}$ zu ver= lieren. $\mathfrak{W a z}$ aber wollen vollendz foldten Gaben bes

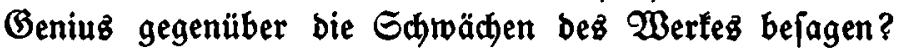
Die Sdjwäden - id fönnte ebenjogut jagen bie 
Ganwäche, benn mit jenem einen, bas Gobineau jelbjt in fpäteren $\mathfrak{\text { abren mit ben }}$ Worten ,il y a trop de conspiration" treffend bezeichnet hat, ift im Grunde alles gefagt. Indem er bies eine Motiv intenî und extenjī zu ftark und zu einjeitig verwertet, hat er fid) fein tra= gijđ)es Gebiet unzweifelhaft fehr verengert. Nur bie Blinbheit beş Ganatiżmuz tönnte biejeź, aber aud nur

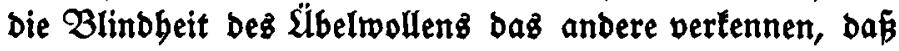
er auf bem alfo verengerten Bebiete nun ed)te tragifhe Größe in Fülle entfaltet hat. Wobl hat Bobineau nur einen 2luz̄jđ)nitt auż einem Riejenbiloe gegeben, aber eż ift ber, ber 2lleranbers Geele voll umífliejt und auz Dem fein Selbenauge, je weniger ez von äuperer Slme gebung zu überfadaen bat, um fo heller unz entgegen= glüht und =leudtet. Die ganze Cinjeitigfeit, aber aud Die ganze Grokgeit ber $\mathfrak{I}$ ugend atmet aus biejem $\mathfrak{W e r k e .}$ $\mathfrak{I n}$ ipäteren $\mathfrak{I a b r e n}$ würbe Bobineau zweifelloz fich etwaz objeftiver gegeben, er würbe vor allem bas (Ganze motivifd reidjer gegliebert, nidt to auf einen Son geftimmt haben. Die Etaffage bätte alazbann zweifellos gewonnen, bie Gejtalten, inbbejondere bie Souptfigur, bagegen febr wabrjđeinlich verloren, wäbrend jegt, wie gerabe bie lebendige 2luffübrung lebren Dürfte, Der Monotonie ber bramatifalen Sauptmotipe mehr als augigleidueno bie meifferbafte Alusmalung ber Sauptgeftalten gegen= überfteht.

Ganz gewwiß hat Gobineau im Aležander mandez mebra al fresco gemalt. Es feblen bie uno ba bie feineren Finzelzüge, bie Nuancierungen unb

1 Go bätte man bie Gelbberrn mehr abgeftuft getwïnidit.

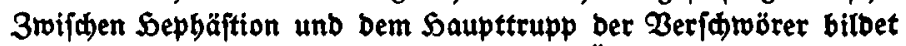
nur allenfalls reard ben fo erwüniकten s̈bergang. 
Febler, ber baburch um fo ftörender wirfen fönnte, weil bie angebeutete Monotonie gerabe bie dez Edhled)ten ift. Indeffen ift er bod) minbeftens nur zum Seil organifa. 3um anbern Seile fann ibm leidst burch ptilifitiche $\mathfrak{d} b$ glättungen und biätrete Wiedergabe abgeholfen werben ${ }^{2}$. Bor allem aber wirb in Gobineaus Ginne immer barauf

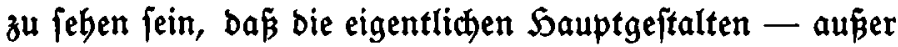
Zllerander felber Rorane, bie Selbin ber Riebe alż (Eifer= fucht, SJephäftion, Der Selb der Freunbjhaft, Perdiffaz, Der Grofmeifter ber Beríchwörerränte -, bie ber Diđhter, wienobl er aud ibre Cbaraftere in Grunbe alle ftart auf einen 3 ag geftellt, babei bod) fo wunberbar lebenzboll zu inbivibualifieren verftanden bat, voll zur Geltung fommen.

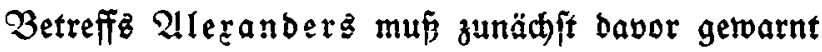
werben, Dás man nidjt etra audi in ber Gailberung bez Gdwantens feiner Gtimmungen feinen Felbherrn gegen. über, wie aud in beren jtarter, faft erzentrifher Eteige: rung uno Färbung, jugendlidge Slnreife finden wolle. Diezz ift vielmebr berwapte Gailberang bes Beniez, baż ja

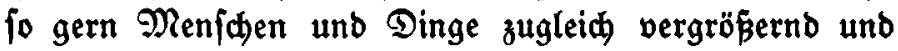

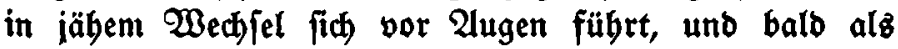
belebenbe, erleudtende, erwärmente Gonne, balb als furd)tbarer $\mathfrak{B u l t a n}$ auf fie einwirtt. Sibrigens befindet fid) gerabe hier (Bobineau im (Fintlange mit ber gef(jid)t= liden Sat fache ber fo erzeffiv veranlagten Indivibualität Ulexanders. (Er fann find nicht genug barin tun, ben

1 Go müffen z. $\mathfrak{B}$. in ber Gđlußjzene, wenn biefe nidht alle ஐürbe verlieren foll, bie Felbberen Durdweg geoämpft, balblaut reben, bis zu Dem 2ugenblife, wo Reonnatus mit erbobener Gtimme bie welthijtorifhe Frage an Uleranber ftellt: wem er feine Rrone vermade. 


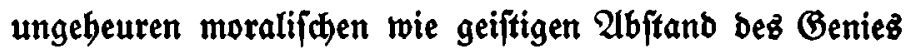
bom Salente barzuftellen, und nur auz ihm ift ber Rontrajt von Aleranders berben Berurteilungen in bäjteren, und bon feinen 2lnnäberungs: = und Emporziebungżver-

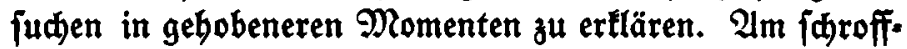
ften tritt biefer Rontraft in feiner legten Anrebe (im zweiten 2uftritt bes legten 2 uffugez) und in bem barauffolgenden Monologe berwor, mag man nun in erferer nur ein turzez janguinifhes 2lufflactern in feiner pejimijtifden Slmbüfterung jeben wollen, ober aunehmen, er habe in ben Blifen ber Feldberrn gelejen, bas er felbjt in einem foldhen lugenbliče von ibnen nicht veritanben worben. Getwi wirb es mandsem in bie Geele fanneiben, Daḱ Gobineau ben welthiftorifaen Moment von ale= tanbers (Enbe in feiner tragifd)en Bitterfeit gar fo grell bingeftellt und beleudftet bat; im ganzen ift ez aber bod fo gewejen: Daz Groß̉e, im böhjiten Ginne genommen, perjđminbet bamit aus biejem Seile ber Welt, auz biejer Epode ber Gefhidhte, unb nur allenfallz nod mandes ₹üdtige vermodte fid in eine neue binüberzuretten, Daz jebod) unjer (Didter bei bem eridütternben 2lbjđiebs. blife,e, ben er von einfamer Säbe in bie Nieberungen wirft, überjeben mujte. Bei einer folden Piebeleere und Beröbung, fold) einem Eieg ber Rleinen würbe uns

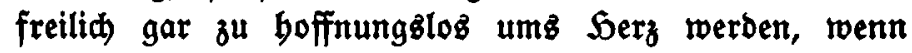
nid)t ber Genius im Gerwanbe edten Gottegignabentums in Bobineaus wunberbarer Daritellung Alleranderz allein (đ)on mit folder Reuchtfraft begabt wäre, Utmofphäre nimmer bunfel werben fann, auch wenn er unterliegt unb untergeht. Der Gonnenglanz von alles

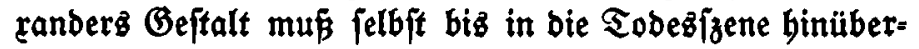
leudten, bie Geelengröß̈e, bie aus feinem legten Mono. 


\section{- XIII -}

loge (prid)t, biefe gleid) einem Gonnenuntergange nadt)= vergolben.

Nur noc) ein $\mathfrak{W o r t}$ über Rorane. Goll biefe Be= ftalt ridtiger 2luffajiung und Darftellung begegnen, jo barf keinen 2ugenbliá vergefifen werben, baß auch fie in allem und jebem einen 3 ug von $\mathfrak{B r a ̈ B z e}$ offenbart. Tank ihm begreift auper Sephäftion einzig fie ben Rönig, auz ibm erjit geminnt aud ibr furchtbarer Rampf zwi jhen Liebe uno (Fiferiud)t jeine wabre tragifa)e Bertiefung. 